Rapid Reviews COVID-19

\title{
Reviews of "Cytotoxic lymphocytes are dysregulated in multisystem inflammatory syndrome in children"
}

Moshe Arditi' ${ }^{1}$, Magali Noval Rivas ${ }^{2}$, Rebecca A. Porritt ${ }^{2}$, Ashraf Harahsheh ${ }^{3}$, Vanessa Bundy ${ }^{4}$, Paolo Palma ${ }^{5}$

${ }^{1}$ Professor, Cedars Sinai Medical Center, Immunology, USA, ${ }^{2}$ Cedars-Sinai Medical Center, ${ }^{3}$ Children's National Hospital, Pediatrics/ Pediatric cardiology, USA,

${ }^{4}$ Division of Allergy and Immunology, Children's National Hospital; The George Washington University School of Medicine \& Health Sciences,

${ }^{5}$ Chilldren's Hospital "Bambino Gesù"

Published on: Nov 22, 2020

DOI: $10.1162 / 2 \mathrm{e} 3983 f 5.948 \mathrm{~b} 17 \mathrm{e} 0$

License: Creative Commons Attribution 4.0 International License (CC-BY 4.0). 
To read the original manuscript, click the link above.

Summary of Reviews: This study compares transcriptomes from patients afflicted with MIS-C, a condition resulting from COVID-19, with other pediatric disorders and finds MIS-C shares a similar molecular etiology to Kawasaki Disease. Reviewers found the claims unsubstantiated and greatly misleading.

Reviewer 1 (Moshe Arditi, Magali Noval Rivas, Rebecca A. Porritt) |

Reviewer 2 (Ashraf Harahsheh, Vanessa Bundy) | $\square \square \square \square$

Reviewer 3 (Paolo Palma)

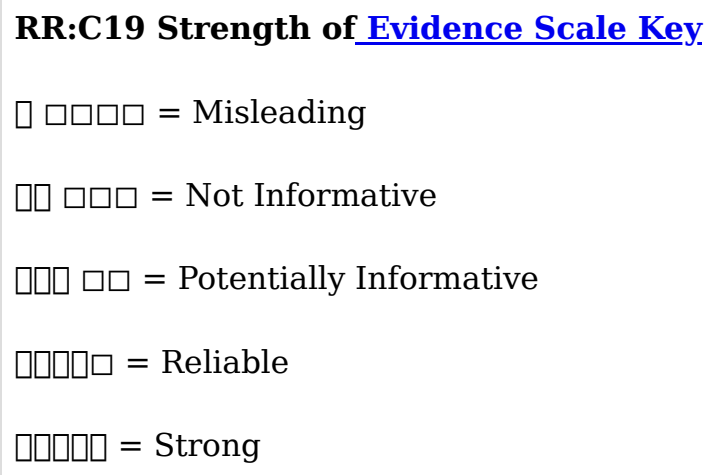

To read the reviews, click the links below. 